\title{
NEDD9 promotes invasion and migration of colorectal cancer cell line HCT116 via JNK/EMT
}

\author{
HAINING MENG ${ }^{1 *}$, JUNYU WU $^{2 *}$, QIAO HUANG ${ }^{1}$, XI YANG $^{1}$, KUNAO YANG $^{1}$, \\ YUEXIN QIU ${ }^{1}$, JIWEN REN $^{1}$, RUOWU SHEN ${ }^{1}$ and HONG QI ${ }^{3}$ \\ ${ }^{1}$ Department of Special Medicine, School of Basic Medical College, Qingdao University, Qingdao, Shandong 266021; \\ ${ }^{2}$ Department of Emergency, First Affiliated Hospital of The People's Liberation Army General Hospital, Beijing 100037; \\ ${ }^{3}$ Department of General Surgery, Qingdao Municipal Hospital, Qingdao, Shandong 266071, P.R. China
}

Received October 9, 2018; Accepted June 6, 2019

DOI: $10.3892 / \mathrm{ol} .2019 .10756$

\begin{abstract}
Neural precursor cell-expressed, developmentally-downregulated 9 (NEDD9) is a multi-domain skeleton protein that serves an important role in the cell signaling process via modulating invasion, metastasis, proliferation and apoptosis of tumor cells. The present study identified that the expression levels of NEDD9 in colorectal cancer were elevated. Therefore, the effect of downregulating the expression of NEDD9 in terms of invasion and migration of colorectal cancer cells was investigated and the role of the JNK pathway in these processes was also investigated. The data revealed that downregulation of NEDD9 and JNK inhibitors suppressed invasion and migration, decreased expression levels of phosphorylated JNK, increased the expression levels of E-cadherin and decreased the expression levels of vimentin. In summary, NEDD9 promotes invasion and migration of colorectal cancer cells via the JNK pathway.
\end{abstract}

\section{Introduction}

Colorectal cancer (CRC) is a common malignant disease that occurs worldwide and is one of the leading causes of cancer-associated mortality in humans $(1,2)$. Although chemotherapeutic and surgical treatments have improved the 5-year survival rate,

Correspondence to: Dr Ruowu Shen, Department of Special Medicine, School of Basic Medical College, Qingdao University, Boya Building, 308 Ningxia Road, Qingdao, Shandong 266021, P.R. China

E-mail: shenruowu@aliyun.com

Professor Hong Qi, Department of General Surgery, Qingdao Municipal Hospital, 5 Donghai Middle Road, Qingdao, Shandong 266071, P.R. China

E-mail: qih99@163.com

*Contributed equally

Key words: neural precursor cell-expressed, developmentallydownregulated 9, c-Jun NH-terminal kinase, EMT
$>50 \%$ of patients present with metastasis at the time of diagnosis, a primary explanation for the high 5-year mortality (3). It is difficult to cure patients with distant metastasis with CRC; therefore, it imperative to identify novel targeted therapy genes (3).

Neural precursor cell-expressed, developmentally-downregulated 9 (NEDD9), also called HEF1 and Cas-L (4), is a multidomain scaffolding protein, belonging to the crk-associated substrate family (5). The most thorough study of NEDD9 suggested that it coordinates adhesion, migration, invasion and cascade reactions of Src and FAK signals (4-7). Numerous studies have reported that NEDD9 modulates invasion and metastasis of gastric cancer (8), breast cancer (9), cervical cancer (6), melanoma (5) and lung cancer (10). We hypothesized that NEDD9 was a biomarker of tumor invasion and metastasis. Previous studies have identified that NEDD9 is expressed in CRC and is closely associated with invasion, metastasis and poor prognosis (11-13). Nevertheless, the specific mechanisms of the effect of NEDD9 on CRC have yet to be completely elucidated.

Epithelial-mesenchymal transition (EMT) is a reversible process of differentiation that causes polarized epithelial cells to lose epithelial characteristics and obtain typical mesenchymal properties, and it has been reported that EMT is closely associated with the progression of malignant tumors (14-16). Studies have demonstrated that NEDD9 promotes tumor invasion and metastasis by activating $\operatorname{EMT}(9,17,18)$. The c-Jun NH-terminal kinase (JNK) is a member of the family of mitogen-activated protein (MAP) kinases (19), and is primarily associated with proliferation, differentiation, apoptosis and migration $(20,21)$. Previous studies have reported that activated JNK promotes the invasion and metastasis of tumors by promoting the development of EMT (22-24).

The aim of the present study was to evaluate whether NEDD9 promoted cell invasion and migration by activating the JNK/EMT signaling pathways in colorectal tumors.

\section{Materials and methods}

Cells and tissues. Colorectal cancer cells HCT116 (Jikai gene, Shanghai, China) and normal colorectal tissue cells FHC (Suzhou Jikai Gene Technology Co., Ltd. Shanghai, China) were cultured in RPMI-1640 with $10 \%$ fetal bovine serum (ExCell Bio, Shanghai, China). Trypsin-EDTA 
(Beijing Solarbio Science and Technology Co., Ltd., Beijing, China) was used to digest cells. Tissue samples were from Qingdao Municipal Hospital between February 2016 and December 2017. Written informed consent was provided by patients. The present study was approved by the Ethics Committee of the Affiliated Hospital of Qingdao University (Qingdao, China). The clinicopathological features including age, sex, stage of Tumor Node Metastasis (25), data and NEDD9 expression status. The inclusion criterion were the following: $<75$ years; clinically proven colorectal cancer tissue and no prior cancer chemotherapy. The exclusion criterion were the following: $\geq 75$ years and prior cancer chemotherapy.

Transfection. The lentiviral downregulation vector (lv-nedd9) (Jikai Gene Chemical Technology Co., Ltd.) and the blank vector (lv-nc) (Jikai Gene Chemical Technology Co., Ltd.) expression of the target gene NEDD9 were constructed. The Lv-NEDD9 and Lv-NC two groups of cells were seeded in 96-well plates at a density of 10,000 cells/well, and a Multiplicity of Infection=25 lentiviral vector and a transfection enhancer Polybrene (Jikai Gene Chemical Technology Co., Ltd.) were added simultaneously $24 \mathrm{~h}$ later. The culture solution was changed after $10 \mathrm{~h}$. The culture was kept for 10 days and then used for experimental research.

Transwell assays. Cells in each group (Lv-NC and Lv-NEDD9) were placed in the Transwell chambers at a density of 30,000 cells/well $(8 \mu \mathrm{m}$, Corning, USA). Serum-free medium with $200 \mu \mathrm{l}$ was added to the upper compartment, and $500 \mu \mathrm{l}$ fetal bovine serum was added to the lower compartment. After $24 \mathrm{~h}$ of culture, the cells in the upper chamber were wiped away. After being fixed with methanol for $10 \mathrm{~min}$ and stained with $0.2 \%$ crystal violet (Solarbio, Beijing, China) for $10 \mathrm{~min}$ at $25^{\circ} \mathrm{C}$, the cells were washed with PBS three times, then images were obtained under an optical microscope (magnification, x400) and counted.

Invasive ability test: Matrigel matrix (Corning Inc.) was mixed with complete medium in a 1:9 ratio. The diluted mixture was added to the Transwell chamber at $100 \mu \mathrm{l} /$ well, in a $37^{\circ} \mathrm{C}$ incubator for $4 \mathrm{~h}$. Cells in each group were placed into Transwell chambers with 50,000 cells/well (8- $\mu \mathrm{m}$, Corning Inc.), and the other steps were the same as the migration experiment.

For HCT116 cell lines, the JNK inhibitor SP600125 (Cell Signaling Technology, Inc.) was dissolved in DMSO and added. This was the experimental group (SP600125 group). By contast, DMSO alone (Beijing Solarbio Science and Technology Co, Ltd.) was the control group (DMSO group). The invasion and metastasis capacities of cells in each were measured by Transwell experiments. The experimental method was carried out as aforementioned.

Wound healing assay. Cells in each group (Lv-NC and Lv-NEDD9) were placed in the six-well plates (Corning, Inc.) at a density of 500,000 cells/well. After $24 \mathrm{~h}$, the cells were scratched with a $200 \mu \mathrm{l}$ sterile pipette tip and photographed at 0 and $24 \mathrm{~h}$. DMSO and SP600125 groups in the scratch test were the same as described earlier.

cDNA synthesis and RT- $q P C R$. Tissue and cell total RNA(Takara, Japan) was extracted using an ISO plus RNA extraction kit (Takara
Table I. Clinicopathological features.

\begin{tabular}{|c|c|c|}
\hline Features & Number & $\begin{array}{l}\text { High expression } \\
\text { of NEDD9 }\end{array}$ \\
\hline \multicolumn{3}{|l|}{ Age (years) } \\
\hline$\geq 50$ & 35 & 30 \\
\hline$<50$ & 5 & 2 \\
\hline \multicolumn{3}{|l|}{ Sex } \\
\hline Female & 29 & 24 \\
\hline Male & 11 & 8 \\
\hline \multicolumn{3}{|l|}{ Grade (TNM) (25) } \\
\hline I & 5 & 2 \\
\hline II & 11 & 8 \\
\hline III & 21 & 19 \\
\hline IV & 3 & 3 \\
\hline \multicolumn{3}{|l|}{ Stage (TNM) (25) } \\
\hline \multicolumn{3}{|l|}{$\mathrm{pT}$} \\
\hline $\mathrm{T} 1$ & 2 & 1 \\
\hline $\mathrm{T} 2$ & 5 & 3 \\
\hline $\mathrm{T} 3$ & 17 & 13 \\
\hline $\mathrm{T} 4$ & 16 & 15 \\
\hline \multicolumn{3}{|l|}{$\mathrm{pN}$} \\
\hline NO & 20 & 14 \\
\hline N1 & 13 & 11 \\
\hline N2 & 7 & 7 \\
\hline \multicolumn{3}{|l|}{$\mathrm{pM}$} \\
\hline M0 & 37 & 29 \\
\hline M1 & 3 & 3 \\
\hline \multicolumn{3}{|c|}{ Date of tissue collection } \\
\hline 02/2016-12/2016 & 12 & 10 \\
\hline 01/2017-12/2017 & 28 & 23 \\
\hline
\end{tabular}

TNM, Tumor Node Metastasis; NEDD9, neural precursor cell-expressed, developmentally-downregulated 9.

Bio, Inc.). A PrimeScript RT reagent kit with gDNA Eraser (Perfect Real Time) was used for reverse transcription (Takara Bio, Inc., Otsu, Japan). SYBR Premix Ex Taq RR420A (Takara Bio, Inc.) was used for quantitative-PCR (RT-qPCR) (Bio-Rad Laboratories, Inc. CFX96) and the thermocycling conditions were as follows: $30 \mathrm{sec}, 1$ cycle, $95^{\circ} \mathrm{C}$ for $5 \mathrm{sec}$, $60^{\circ} \mathrm{C}$ for $30 \mathrm{sec}, 40$ cycles. The expression levels were analysed by $2^{-\Delta \Delta \mathrm{Cq}}$ method (26). Primers used in the experiment were purchased from Shanghai Sangon Biotech. NEDD9: Forward: 5'-GAGCTGGATGGATGACTACGA-3'; Reverse: 5'-AGCTCT TTCTGTTGCCTCTCA-3'. E-cadherin: Forward: 5'-CTGATT CTGCTGCTCTTGCTG-3'; Reverse: 5'-CTTCTCCGCCTC CTTCTTCA-3'. Vimentin: Forward: 5'-GAAATTGCAGGA GGAGATGC-3'; Reverse: 5'-ATTCCACTTTGCGTTCAA GG-3'. $\beta$-actin: Forward: 5'-CACCATGAAGATCAAGATCAT TGC-3'; Reverse: 5'-GGCCGGACTCATCGTACTCCTGC-3'.

Western blot analysis. The four groups cells, Lv-NEDD9, Lv-NC, DMSO and SP600125 (3 ml $50 \mu \mathrm{M}$ SP600125 for 

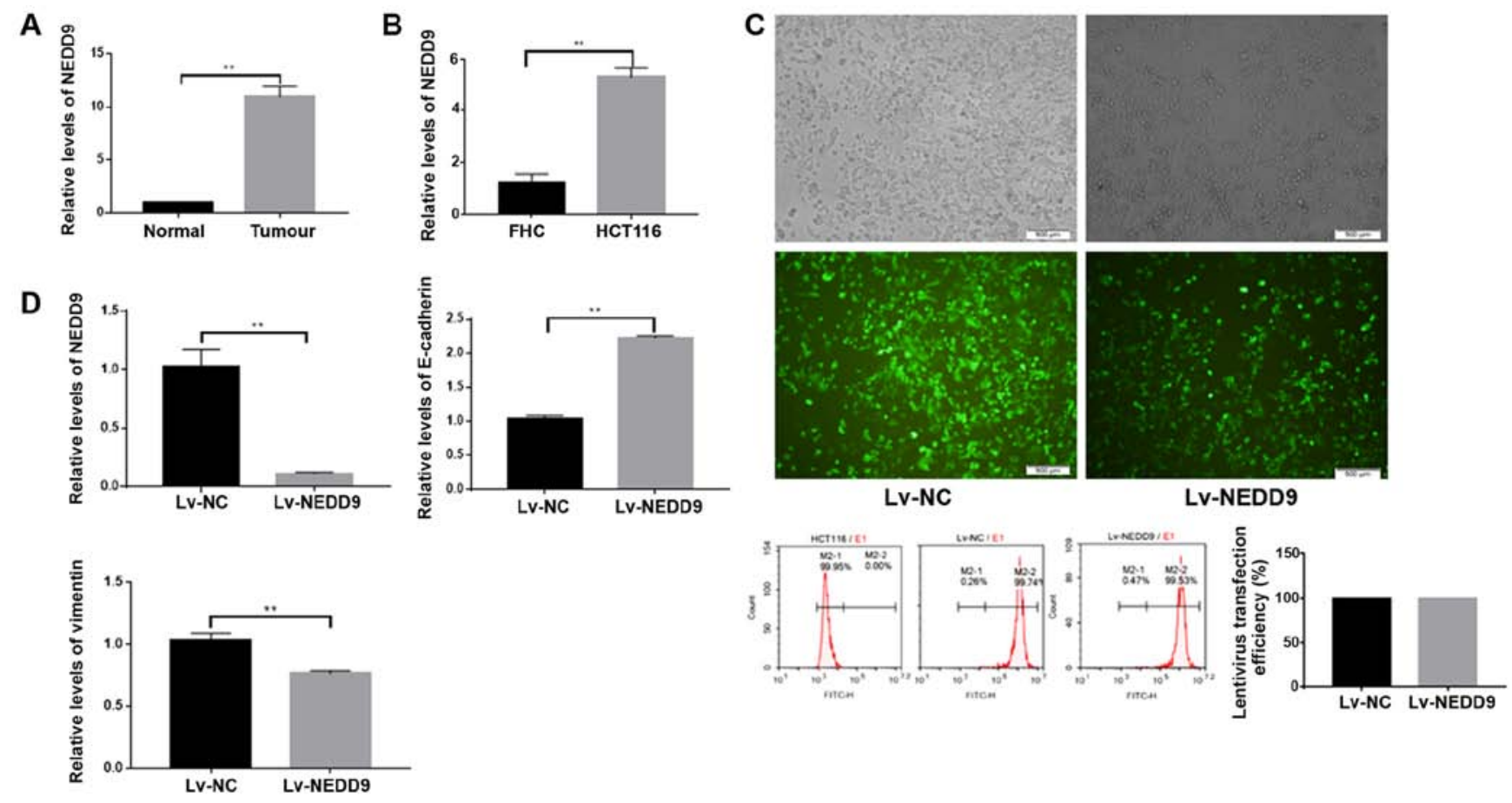

Figure 1. Expression levels of NEDD9 were upregulated in CRC tissues and CRC cell lines. Changes of EMT markers following down regulation of NEDD9. (A) The relative levels of NEDD9 in CRC tissues and normal colorectal tissues. (B) The relative levels of NEDD9 in the FHC and HCT116 cell lines. (C) Effect of lentiviral transfection in HCT116 cell lines. Scale bar=500 $\mu \mathrm{m}$. (D) Changes of NEDD9, E-cadherin and vimentin following transfection in HCT116 cells. ${ }^{* *} \mathrm{P}<0.01$. CRC, colorectal cancer; NEDD9, neural precursor cell-expressed, developmentally-downregulated 9; Lv, lentivirus; NC, negative control.

$5 \mathrm{~h}$ at $37^{\circ} \mathrm{C}$ ), were washed twice with PBS and $1 \mathrm{ml}$ PBS was added. The cells in the culture flask were scraped off with a cell scalpel and centrifuged for $5 \mathrm{~min}$ at $4^{\circ} \mathrm{C}$ at $5,000 \mathrm{x} \mathrm{g}$. Following removal of the supernatant, RIPA lysis buffer (Beijing Kangwei Century Biotechnology Co., Ltd.) was used to extract whole protein. After adding protease and phosphatase inhibitors, proteins were placed on ice for $15 \mathrm{~min}$. After vortexing, proteins were again placed on ice for $15 \mathrm{~min}$. The lysates were centrifuged at $12,000 \mathrm{x}$ g for $10 \mathrm{~min}$. The supernatants contained total protein, the protein determination (Thermo Fisher Scientific, Inc.) method was the BCA method. The extracted protein was mixed with the sample buffer SDS-loading buffer, and boiled for $5 \mathrm{~min}$. A total of $15 \mu \mathrm{l}$ of protein was loaded per lane. Samples were separated by $10 \%$ gel electrophoresis. Proteins were then transferred to PVDF membranes (EMD Millipore). The membranes were blocked in 5\% skim milk for $1 \mathrm{~h}$ at room temperature. NEDD9, JNK, $\beta$-actin, E-cadherin and vimentin antibodies were incubated overnight at $4^{\circ} \mathrm{C}$. The enhanced chemiluminescent substrate reagent (ECL) was applied to the film and was analyzed on a Quant LAS 4010 imaging system (Ultra-Violet Products Ltd.).

The antibodies used in this experiment were as follows: The primary antibodies were 1:1,000 mouse anti-NEDD9 (cat. no., 4044, CST); 1:1,000 rabbit anti-JNK (cat. no., 9252, CST); 1:5,000 rabbit anti- $\beta$-actin (cat. no., bs-0061R, BIOSS, Beijing, China); 1:1,000 rabbit anti-E-cadherin (cat. no., 3195, CST); and 1:1,000 rabbit anti-vimentin (cat. no., 5741, CST). All primary antibodies were treated at $4^{\circ} \mathrm{C}$ for $12 \mathrm{~h}$. The secondary antibodies were 1:1,000 [cat. no., ab6708, goat anti-mouse IgG H\&L (HRP), Abcam] or 1:1,500 (cat. no., bs-0295G, goat anti-rabbit IgG, BIOSS), and incubated for $2 \mathrm{~h}$ at $25^{\circ} \mathrm{C}$.
Statistical analysis. GraphPad Prism 7 software (GraphPad Software, Inc., La Jolla, CA, USA) was used for statistical analysis. The results of the experiments are presented as the means with standard deviations. Data were compared using the paired t-test. $\mathrm{P}<0.05$ was considered to indicate a statistically significant difference.

\section{Results}

NEDD9 was highly expressed in colorectal cancer. First, the present study measured expression levels of NEDD9 in 40 normal colorectal tissues and colorectal cancer tissues by RT-qPCR, and observed that NEDD9 was highly expressed (Fig. 1A and Table I) in tumor tissues. Subsequently, the expression levels of NEDD9 were measured in the normal colorectal cell line FHC and CRC cell line HCT116, and identified that NEDD9 was also highly expressed in the CRC cell line (Fig. 1B).

Expression of EMT changed following the downregulation of NEDD9. The present study downregulated NEDD9 in colorectal cancer cells using lentiviral transfection, and observed that the transfection efficacy was $>90 \%$ ( $\mathrm{P}<0.01$; Fig. 1C). NEDD9 expression in the Lv-NEDD9 group was significantly lower than in the $\mathrm{Lv}-\mathrm{NC}$ group as measured by RT-qPCR (P<0.01; Fig. 1D). The E-cadherin expression levels were significantly higher in the Lv-NEDD9 group than that in the Lv-NC group, and the vimentin expression levels were significantly lower in Lv-NEDD9 group when compared with the Lv-NC group ( $\mathrm{P}<0.01$; Fig. 1D). These data suggest that the expression of EMT markers was altered following NEDD9 downregulation. 
A

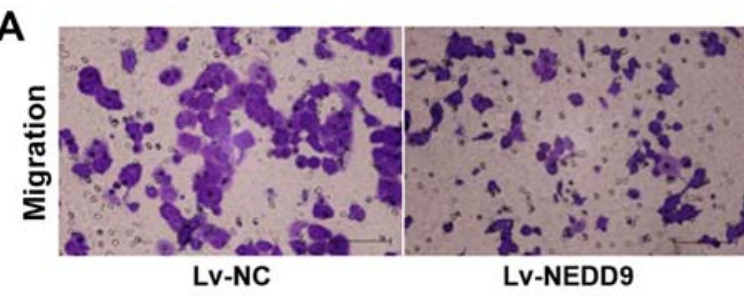

B
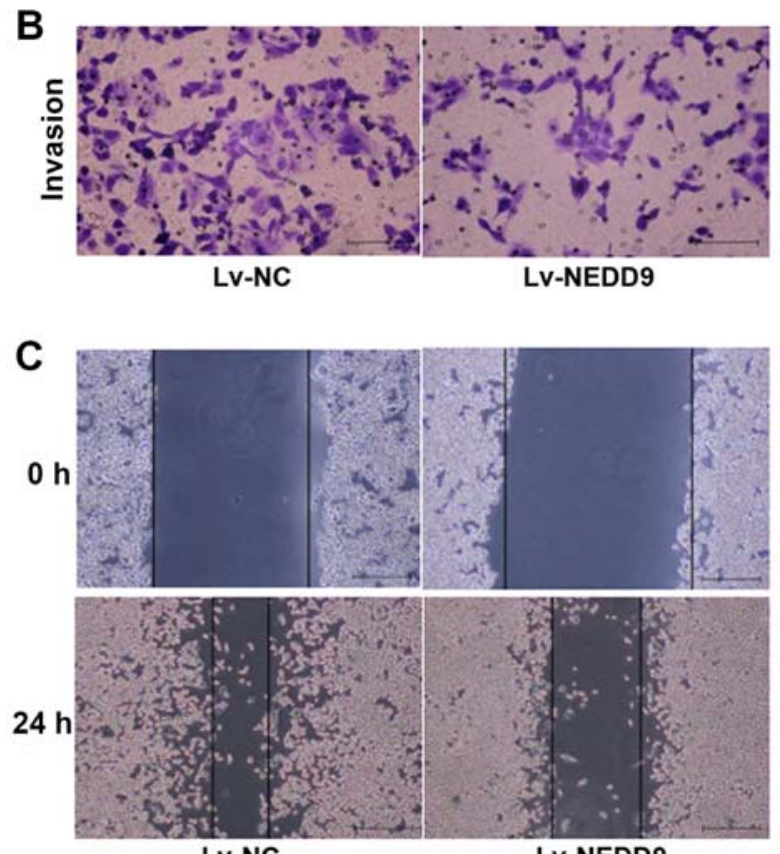
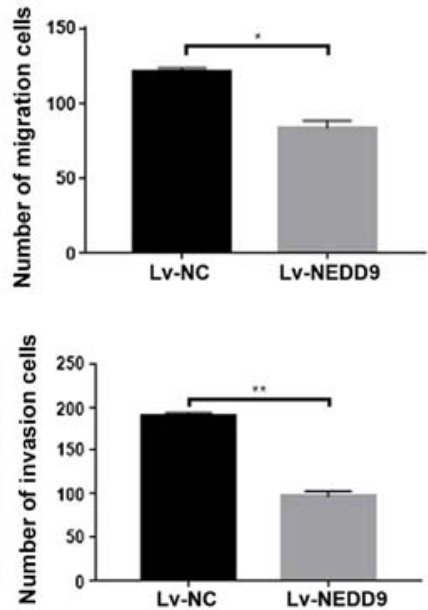

LV-NC Lv-NEDD9

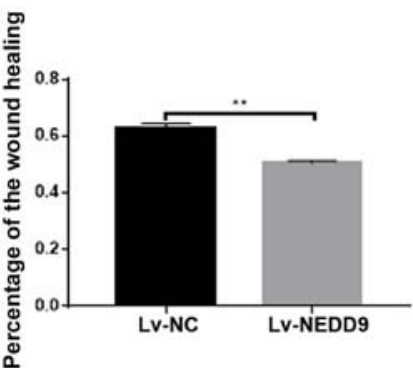

Figure 2. Downregulated NEDD9 inhibited the migration and invasion levels of HCT116 cells. (A) The results of migration assays for HCT116 cells following transfection. Scale bar $=100 \mu \mathrm{m}$. (B) The results of invasion assays for HCT116 cells following transfection. Scale bar=100 $\mu \mathrm{m}$. (C) The results of wound healing assays in HCT116 cells following transfection. Scale bar $=400 \mu \mathrm{m}$. ${ }^{*} \mathrm{P}<0.05,{ }^{* *} \mathrm{P}<0.01$. NEDD9, neural precursor cell-expressed, developmentally-downregulated 9; Lv, lentivirus; NC, negative control.
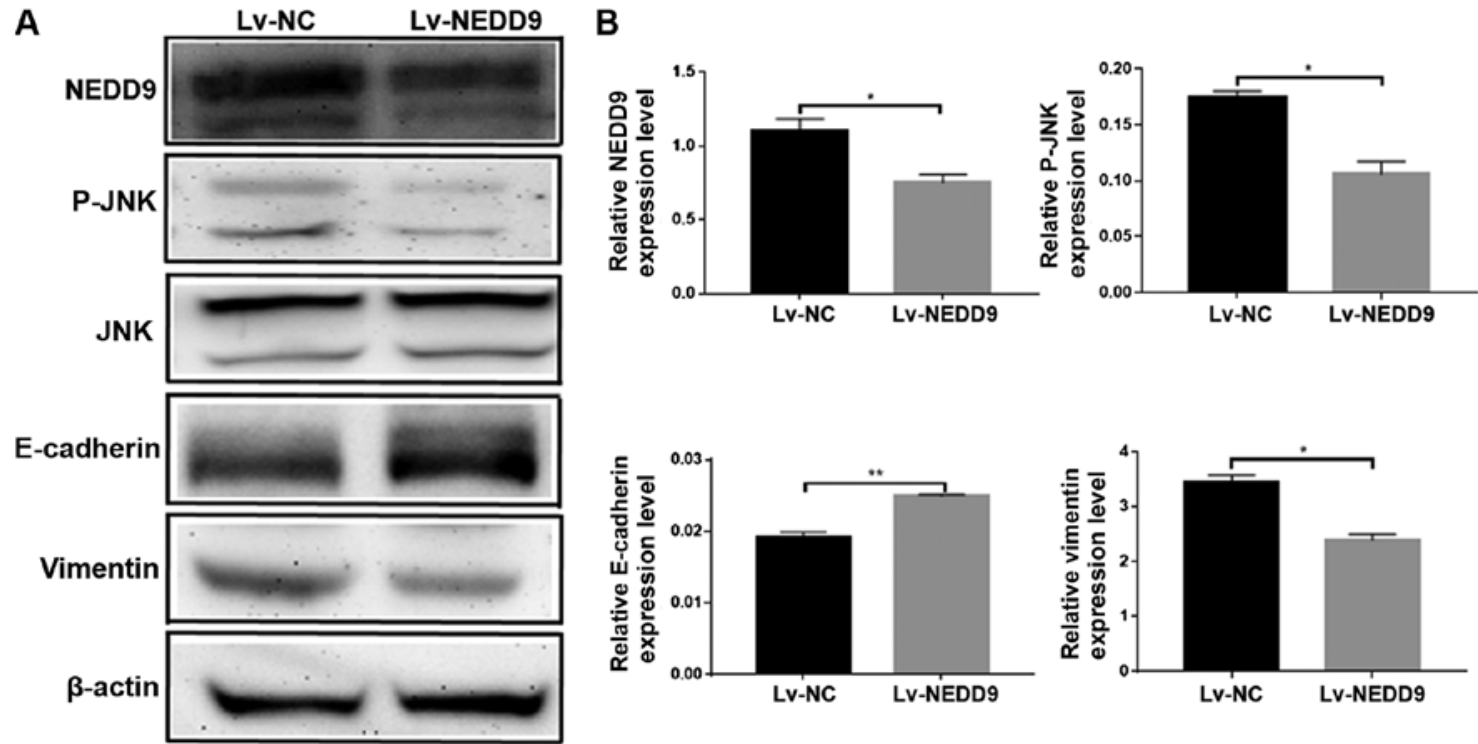

Figure 3. Downregulated NEDD9 inhibited expression levels of JNK and suppressed EMT. (A) Western blot assays for NEDD9, E-cadherin and vimentin in HCT116 cells after transfection. (B) The analysis for the results of western blots. ${ }^{*} \mathrm{P}<0.05,{ }^{* *} \mathrm{P}<0.01$. NEDD9, neural precursor cell-expressed, developmentally-downregulated $9 \mathrm{Lv}$, lentivirus; NC, negative control.

Attenuation of the ability of invasion and migration ability of colorectal cancer cells following downregulation of NEDD9.
Cell migration ability $(\mathrm{P}<0.05$; Fig. $2 \mathrm{~A})$ and invasion $(\mathrm{P}<0.01$; Fig. 2B) was significantly lower in the Lv-NEDD9 group than 

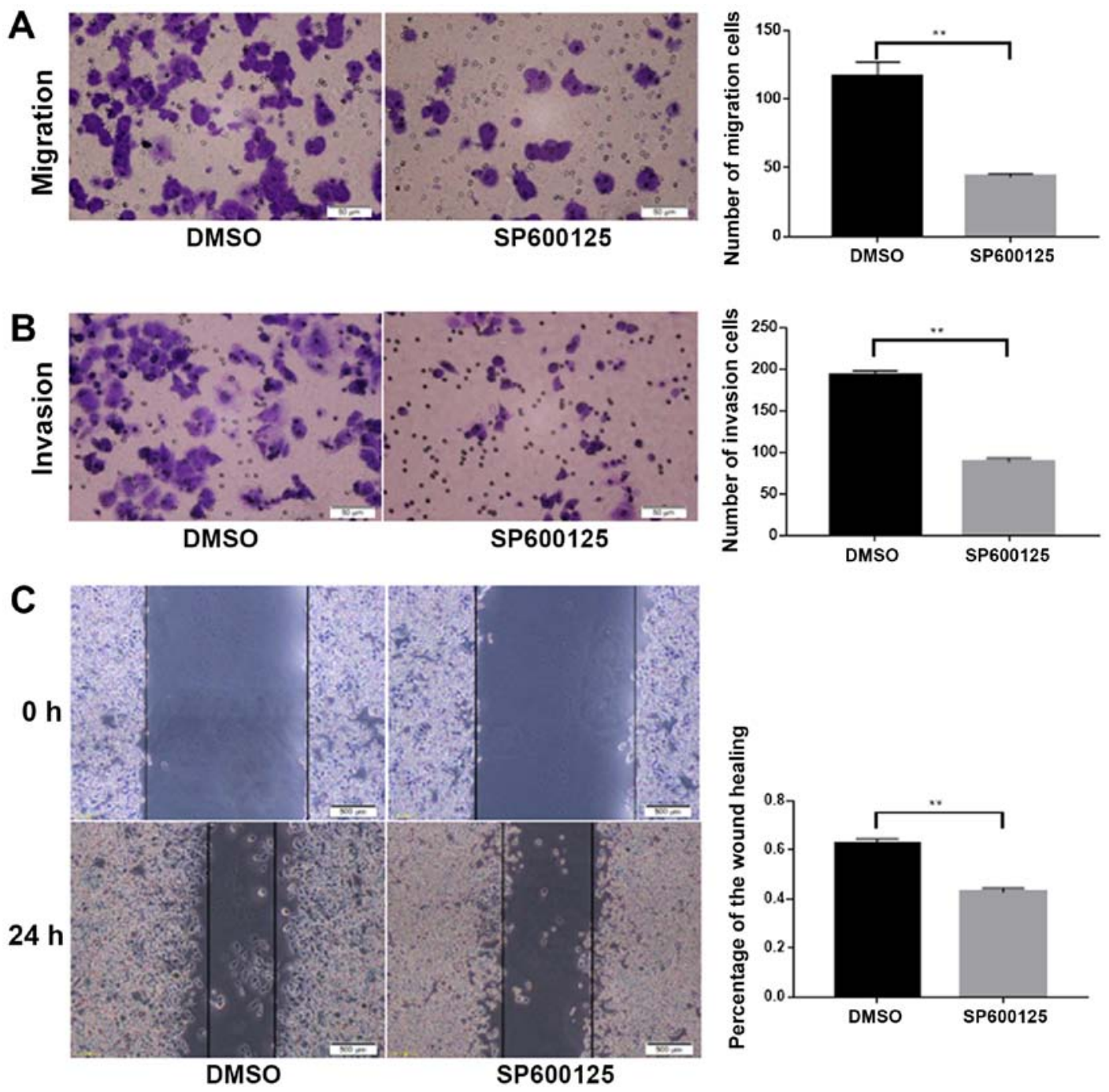

Figure 4. JNK promoted migration and invasion in HCT116 cells. (A) The results of migration assays for HCT116 cells following the addition of SP600125. Scale bar $=100 \mu \mathrm{m}$. (B) The results of invasion assays for HCT116 cells following the addition of SP600125. Scale bar=100 $\mu \mathrm{m}$. (C) The results of wound healing assays for HCT116 cells prior to, and following the addition of SP600125. Scale bar $=400 \mu \mathrm{m} .{ }^{* *} \mathrm{P}<0.01$.

that in the Lv-NC group. The wound healing assay results demonstrated that cell migration $(\mathrm{P}<0.01$; Fig. $2 \mathrm{C})$ was significantly lower in the Lv-NEDD9 group than that in the Lv-NC group. These data suggested that downregulation of NEDD9 inhibits the invasion and migration of colorectal cancer.

NEDD9 regulates the expression of JNK/EMT in HCT116 cell lines. The present study performed western blot analysis to measure the expressions of JNK, E-cadherin and vimentin following the downregulation of NEDD9. The results revealed that the protein expression levels of NEDD9 were decreased, the expression levels of p-jnk were decreased, the expression levels of E-cadherin were increased and the expression levels of vimentin were decreased (Fig. 3B). E-cadherin and Vimentin are EMT-related proteins (27), and the changes of the expressions of JNK, E-cadherin and Vimentin suggest that NEDD9 modulates invasion and migration of CRC by regulating JNK and EMT.

JNK inhibitor SP600125 suppressed invasion and migration of colorectal cancer cells. To investigate whether NEDD9 affected the invasion and migration of colorectal cancer through the JNK pathway, the JNK inhibitor SP600125 was dissolved in DMSO and added to HCT116 cells. Migration $(\mathrm{P}<0.01$; Fig. 4A) and invasion $(\mathrm{P}<0.01$; Fig. 4B) were significantly lower in the SP600125 group than in the DMSO group. Wound healing assays demonstrated that migration was significantly lower in the SP600125 group when compared with the DMSO group ( $\mathrm{P}<0.01$; Fig. 4C). Taken together, these data suggested that JNK promoted the invasion and migration abilities of colorectal cancer.

JNK inhibitor SP600125 delayed the progression of EMT. Following the addition of the JNK inhibitor SP600125, changes in expression levels of the EMT-related proteins E-cadherin and vimentin were examined by western blotting (Fig. 5A). E-cadherin expression levels increased and the vimentin expression levels were decreased (Fig. 5B). These data suggested that JNK promoted EMT. Taken together, the results of the present study demonstrated that NEDD9 regulates EMT by JNK to promote CRC invasion and migration. 
A

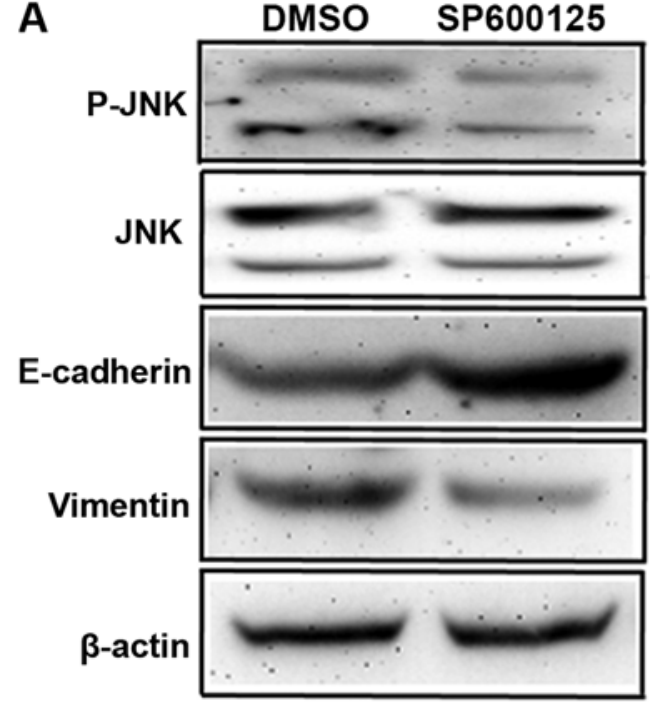

B
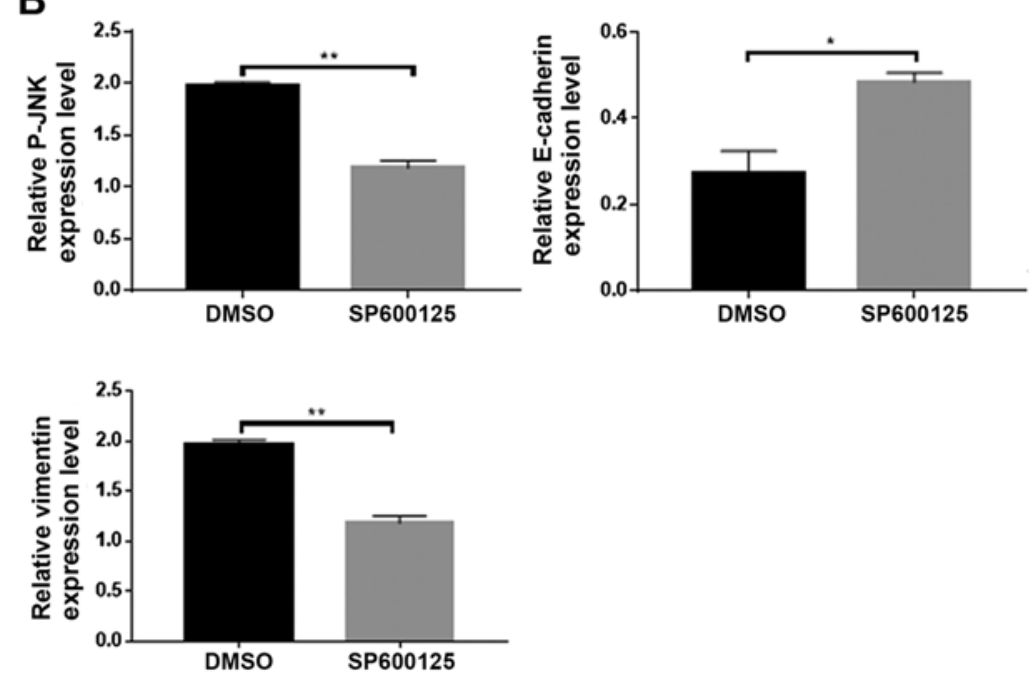

Figure 5. JNK promoted EMT. (A) Western blot assays for JNK, E-cadherin and vimentin in HCT116 after adding SP600125. (B) Quantification of the western blot results following the addition of SP600125. ${ }^{*} \mathrm{P}<0.05,{ }^{* *} \mathrm{P}<0.01$. EMT, epithelial mesenchymal transition.

\section{Discussion}

Several studies have demonstrated that NEDD9 is highly expressed in tumors and functions as an oncogene (4). For example, NEDD9 promotes invasion and migration of melanoma (7) and modulates the metastatic ability of gastric cancer (8), the invasion ability of breast (9) and the migration and invasion ability of cervical cancer (6), which was consistent with the findings of the present study. The present study observed that NEDD9 was highly expressed in CRC and promoted invasion and migration of colorectal cancer cells. The current literature indicates that the majority of studies on NEDD9 focus on its influence on tumor invasion and migration.

NEDD9 acts as a tumor-promoting factor, and its high expression in tumors also promotes the development of EMT $(9,28,29)$. NEDD9 promoted EMT through the ERK pathway in breast cancer (9), as well as promoting EMT by inhibiting Smad7 in liver cancer (18), and promoting metastasis of gastric cancer cells by regulating EMT in gastric cancer (30). The experimental data of the present study demonstrated that the downregulation of NEDD9 in colorectal cancer increased the expression of the EMT-related protein E-cadherin and decreased the expression of vimentin, suggesting that the downregulation of NEDD9 can attenuate EMT. For example, TGF- $\beta 1$ triggers EMT in lung cancer via the JNK pathway (31). Inhibition of JNK reversed EMT and inhibited the migration of gastric cancer (32). JNK has also been reported to promote EMT in renal cell carcinoma (33). These data are consistent with our experimental hypothesis. The present study downregulated NEDD9 and observed that the expression of p-JNK was decreased and the EMT-related protein expression changed. Therefore, it was hypothesized that NEDD9 may regulate the development of EMT via the JNK signaling pathway in CRC. Therefore, the JNK inhibitor SP600125 was added to colorectal cancer cells and the expression of EMT-related proteins was measured. The results revealed that the expression of EMT-related proteins changed.
Taken together the results of the present study demonstrated that NEDD9 promotes EMT via the JNK pathway in colorectal cancer.

NEDD9 mediated tumor invasion by promoting the secretion of MMP9 (34), and the negative regulation of tumor suppressor gene LKB1 modulated the progression of lung cancer (35). NEDD9 is also affected by miRNA regulation affecting tumor invasion and metastasis (36). NEDD9 as a tumor factor is closely associated with the occurrence and development of tumors (37); nevertheless, numerous mechanisms for NEDD9 are unknown and have not been studied; therefore, the current state of knowledge of NEDD9 remains scarce and requires further investigation.

Consult the relevant literature to know that NEDD9 can interact with FAK to affect tumors (38) and related literature has also found that FAK can activate JNK (39), so NEDD9 may activate JNK through FAK. In addition, NEDD9 may also activate JNK via P38MAPK-Erk1/2 (40). NEDD9 may also activate JNK in other ways, but it has not been discovered yet. A review of the literature reveals the relevant situation downstream of JNK. For example, IL-2/sorafenib can affect tumor survival, growth and mobility through JNK-TAZ pathways (41), GPS1 can activate the c-Jun N-terminal kinase (JNK)/Jun pathway to affect breast cancer growth and migration (42), Saikosaponin D induced apoptosis via the activation of TGF $\alpha$-JNK-p53 (43), Thymoquinone inhibits the metastasis through activation of JNK-p38 (44), JNK-ELK1 The pathway can also have an effect on the tumor (45). These are the pathways already known downstream of JNK, but there must be downstream pathways that have not been studied.

Our experiment investigated the effect of NEDD9 on colorectal cancer through JNK, which is currently not done in colorectal cancer. It is innovative and meaningful. However, due to the limitations of experimental conditions, we did not study the specific mechanism of NEDD9 activation of JNK and the downstream pathway of JNK in this experiment. In future experiments, we will work to study the specific mechanism by 
which NEDD9 activates JNK and the pathway downstream of JNK.

In conclusion, we found that NEDD9 was highly expressed in colorectal cancer tissues and colorectal cancer cell lines, and that NEDD9 promoted invasion and migration of colorectal cancer. These data suggested that NEDD9 can be used as a new and effective target for therapy of colorectal cancer.

\section{Acknowledgements}

Not applicable.

\section{Funding}

No funding was received.

\section{Availability of data and materials}

All data generated or analyzed are included in this published article.

\section{Authors' contributions}

$\mathrm{HM}$ and RS were responsible for study design, the acquisition and analysis of data. HM, JW performed the acquisition of data, and wrote and revised the manuscript. JW, QH, XY, KY, YQ and HQ aided with the acquisition of data. HM and JW aided with the statistical analysis. RS and HQ participated in the design and coordination of the study and helped modify the manuscript. JR acquired the data, wrote and revised the manuscript, and helped to perform the statistical analysis. All authors read and approved the final manuscript.

\section{Ethics approval and consent to participate}

This study was approved by the Ethics Committee of the Affiliated Hospital of Qingdao University (Qingdao, China). Witten informed consent was obtained from the patients.

\section{Patient consent for publication}

Not applicable.

\section{Competing interests}

The authors declare that they have no competing interests.

\section{References}

1. Siegel RL, Miller KD, Fedewa SA, Ahnen DJ, Meester RGS Barzi A and Jemal A: Colorectal cancer statistics, 2017. CA Cancer J Clin 67: 177-193, 2017.

2. Siegel R, Desantis C and Jemal A: Colorectal cancer statistics, 2014. CA Cancer J Clin 64: 104-117, 2014.

3. Mcquade RM, Stojanovska V, Bornstein JC and Nurgali K: Colorectal cancer chemotherapy: The evolution of treatment and new approaches. Curr Med Chem 24: 1537-1557, 2017.

4. Izumchenko E, Singh MK, Plotnikova OV, Tikhmyanova N, Little JL, Serebriiskii IG, Seo S, Kurokawa M, Egleston BL, Klein-Szanto A, et al: NEDD9 promotes oncogenic signaling in mammary tumor development. Cancer Res 69: 7198-7206, 2009.

5. Kim M, Gans JD, Nogueira C, Wang A, Paik JH, Feng B, Brennan C, Hahn WC, Cordon-Cardo C, Wagner SN, et al: Comparative oncogenomics identifies NEDD9 as a melanoma metastasis gene. Cell 125: 1269-1281, 2006.
6. Sima N, Cheng X, Ye F, Ma D, Xie X and Lü W: The overexpression of scaffolding protein NEDD9 promotes migration and invasion in cervical cancer via tyrosine phosphorylated FAK and SRC. PLoS One 8: e74594, 2013.

7. O'Neill G, Seo S, Serebriiskii IG, Lessin SR and Golemis EA: A new central scaffold for metastasis: Parsing HEF1/Cas-L/NEDD9. Cancer Res 67: 8975-8979, 2007.

8. Zhang S, Wu L, Liu Q, Chen K and Zhang X: Elevated expression of NEDD9 is associated with metastatic activity in gastric cancer. Onco Targets Ther 8: 633-640, 2015.

9. Kong C, Wang C, Wang L, Ma M, Niu C, Sun X, Du J, Dong Z, Zhu S, Lu J and Huang B: NEDD9 is a positive regulator of epithelial-mesenchymal transition and promotes invasion in aggressive breast cancer. PLoS One 6: e22666, 2011.

10. Chang JX, Gao F, Zhao GQ and Zhang GJ: Expression and clinical significance of NEDD9 in lung tissues. Med Oncol 29: 2654-2660, 2012.

11. Cui X, Shen K, Xie Z, Liu T and Zhang H: Identification of key genes in colorectal cancer using random walk with restart. Mol Med Rep 15: 867-872, 2017

12. Li P, Zhou H, Zhu X, Ma G, Liu C, Lin B and Mao W: High expression of NEDD9 predicts adverse outcomes of colorectal cancer patients. Int J Clin Exp Pathol 7: 2565-2570, 2014.

13. Dai J, Van Wie PG, Fai LY, Kim D, Wang L, Poyil P, Luo J and Zhang Z: Downregulation of NEDD9 by apigenin suppresses migration, invasion, and metastasis of colorectal cancer cells. Toxicol Appl Pharmacol 311: 106-112, 2016.

14. Thiery JP: Epithelial-mesenchymal transitions in tumour progression. Nat Rev Cancer 2: 442-454, 2002.

15. Kalluri R and Weinberg RA: The basics of epithelial-mesenchymal transition. J Clin Invest 119: 1420-1428, 2009.

16. Min J, Liu L, Li X, Jiang J, Wang J, Zhang B, Cao D, Yu D, Tao D, Hu J, et al: Absence of DAB2IP promotes cancer stem cell like signatures and indicates poor survival outcome in colorectal cancer. Sci Rep 5: 16578, 2015.

17. Guerrero MS, Parsons JT and Bouton AH: Cas and NEDD9 contribute to tumor progression through dynamic regulation of the cytoskeleton. Genes Cancer 3: 371-381, 2012.

18. Wang Z, Shen M, Lu P, Li X, Zhu S and Yue S: NEDD9 may regulate hepatocellular carcinoma cell metastasis by promoting epithelial-mesenchymal-transition and stemness via repressing Smad7. Oncotarget 8: 1714-1724, 2017.

19. Weston CR and Davis RJ: The JNK signal transduction pathway. Curr Opin Genet Dev 12: 14-21, 2002.

20. Wagner EF and Nebreda AR: Signal integration by JNK and p38 MAPK pathways in cancer development. Nat Rev Cancer 9: 537-549, 2009.

21. Bode AM and Dong Z: The functional contrariety of JNK. Mol Carcinog 46: 591-598, 2007.

22. Zhao X, Wu X, Qian M, Song Y, Wu D and Zhang W: Knockdown of TGF- $\beta 1$ expression in human umbilical cord mesenchymal stem cells reverts their exosome-mediated EMT promoting effect on lung cancer cells. Cancer Lett 428: 34-44, 2018.

23. Wang Y, Xu Y, Yan W, Han P, Liu J, Gong J, Li D, Ding X, Wang H, Lin Z, et al: CFIm25 inhibits hepatocellular carcinoma metastasis by suppressing the $\mathrm{p} 38$ and JNK/c-Jun signaling pathways. Oncotarget 9: 11783-11793, 2018.

24. Dong Y, Wu Z, He M, Chen Y, Chen Y, Shen X, Zhao X, Zhang L, Yuan B and Zeng Z: ADAM9 mediates the interleukin-6-induced Epithelial-Mesenchymal transition and metastasis through ROS production in hepatoma cells. Cancer Lett 421: 1-14, 2018.

25. Edge SB and Compton CC: The American Joint Committee on Cancer: The 7th edition of the AJCC cancer staging manual and the future of TNM. Ann Surg Oncol 17: 1471-1474, 2010.

26. Livak KJ and Schmittgen TD: Analysis of relative gene expression data using real-time quantitative PCR and the 2(-Delta Delta C(T)) method. Methods 25: 402-408, 2001.

27. Scanlon CS, Van Tubergen EA, Inglehart RC and D'Silva NJ: Biomarkers of epithelial-mesenchymal transition in squamous cell carcinoma. J Dent Res 92: 114-121, 2013.

28. Morimoto K, Tanaka T, Nitta Y, Ohnishi K, Kawashima H and Nakatani T: NEDD9 crucially regulates TGF- $\beta$-triggered epithelial-mesenchymal transition and cell invasion in prostate cancer cells: Involvement in cancer progressiveness. Prostate 74: 901-910, 2014.

29. Miao Y, Li AL, Wang L, Fan CF, Zhang XP, Xu HT, Yang LH, Liu Y and Wang EH: Overexpression of NEDD9 is associated with altered expression of E-cadherin, $\beta$-catenin and $\mathrm{N}$-cadherin and predictive of poor prognosis in non-small cell lung cancer. Pathol Oncol Res 19: 281-286, 2013. 
30. Feng J, Zhao J, Xie H, Yin Y, Luo G, Zhang J, Feng Y and Li Z: Involvement of NEDD9 in the invasion and migration of gastric cancer. Tumour Biol 36: 3621-3628, 2015.

31. Khan GJ, Gao Y, Gu M, Wang L, Khan S, Naeem F, Semukunzi H, Roy D, Yuan S and Sun L: TGF- $\beta 1$ Causes EMT by regulating $\mathrm{N}$-Acetyl glucosaminyl transferases via downregulation of non muscle myosin II-A through JNK/P38/PI3K pathway in lung cancer. Curr Cancer Drug Targets 18: 209-219, 2018.

32. Choi Y, Ko YS, Park J, Choi Y, Kim Y, Pyo JS, Jang BG, Hwang DH, Kim WH and Lee BL: HER2-induced metastasis is mediated by $\mathrm{AKT} / \mathrm{JNK} / \mathrm{EMT}$ signaling pathway in gastric cancer. World J Gastroenterol 22: 9141-9153, 2016.

33. An J, Guo Y, Wang T, Pantuck AJ and Rettig MB: Pomegranate extract inhibits EMT in clear cell renal cell carcinoma in a NF- $\kappa$ B and JNK dependent manner. Asian J Urol 2: 38-45, 2015.

34. Grauzam S, Brock AM, Holmes CO, Tiedeken JA, Boniface SG, Pierson BN, Patterson DG, Coaxum SD, Neskey DM and Rosenzweig SA: NEDD9 stimulated MMP9 secretion is required for invadopodia formation in oral squamous cell carcinoma. Oncotarget 9: 25503-25516, 2018.

35. Feng Y, Wang Y, Wang Z, Fang Z, Li F, Gao Y, Liu H, Xiao T, Li F, Zhou Y, et al: The CRTC1-NEDD9 signaling axis mediates lung cancer progression caused by LKB1 loss. Cancer Res 72: 6502-6511, 2012.

36. Chu Q, Zhang $\mathbf{J}$ and Sun F: Study on mechanism of mi R-203 inhibiting migration and invasion of breast cancer cell MDA-MB-231 by regulating NEDD9 protein. J Diagnostics Concepts Pract, 2014.

37. Shagisultanova E, Gaponova AV, Gabbasov R, Nicolas E and Golemis EA: Preclinical and clinical studies of the NEDD9 scaffold protein in cancer and other diseases. Gene 567: 1-11, 2015.

38. Guerrero MS, Parsons JT and Bouton AH: Cas and NEDD9 contribute to tumor progression through dynamic regulation of the cytoskeleton. Genes Cancer 3: 371-381, 2012.
39. Sulzmaier FJ, Jean C and Schlaepfer DD: FAK in cancer: Mechanistic findings and clinical applications. Nat Rev Cancer 14: 598-610, 2014.

40. Tikhmyanova N, Little JL and Golemis EA: CAS proteins in normal and pathological cell growth control. Cell Mol Life Sci 67: 1025-1048, 2010.

41. Ding X, Sun W and Chen J: IL-2 augments the sorafenib-induced apoptosis in liver cancer by promoting mitochondrial fission and activating the JNK/TAZ pathway. Cancer Cell Int 18: 176, 2018.

42. Li Z, Lim SK, Liang X and Lim YP: The transcriptional coactivator WBP2 primes triple-negative breast cancer cells for responses to Wnt signaling via the JNK/Jun kinase pathway. J Biol Chem 293: 20014-20028, 2018.

43. Chen X, Liu C, Zhao R, Zhao P, Wu J, Zhou N and Ying M: Synergetic and antagonistic molecular effects mediated by the feedback loop of p53 and JNK between Saikosaponin D and SP600125 on lung cancer A549 cells. Mol Pharm 15: 4974-4984, 2018.

44. Kubala MH, Punj V, Placencio-Hickok VR, Fang H, Fernandez GE, Sposto R and DeClerck YA: Plasminogen activator Inhibitor-1 promotes the recruitment and polarization of macrophages in cancer. Cell Rep 25: 2177-2191.e7, 2018.

45. Hu Z, Tie Y, Lv G, Zhu J, Fu H and Zheng X: Transcriptional activation of miR-320a by ATF2, ELK1 and YY1 induces cancer cell apoptosis under ionizing radiation conditions. Int J Oncol 53: 1691-1702, 2018.

(i) $(9)$ This work is licensed under a Creative Commons Attribution-NonCommercial-NoDerivatives 4.0 International (CC BY-NC-ND 4.0) License. 